\title{
THE LINEAR AND QUADRATIC SCHUR SUBGROUPS OVER THE $S$-INTEGERS OF A NUMBER FIELD
}

\author{
CARL R. RIEHM
}

(Communicated by Warren J. Wong)

\begin{abstract}
Let $K$ be an algebraic number field and let $\mathcal{D}$ be a ring of $S$ integers in $K$ (where $S$ is a set of primes of $K$ containing all the archimedean primes); that is to say, $\mathfrak{D}$ is a Dedekind domain whose field of quotients is $K$. In analogy with a theorem of $\mathrm{T}$. Yamada in the case of a field of characteristic 0 , it is shown that if $S(\mathfrak{D})$ is the Schur subgroup of the Brauer group $B(\mathfrak{D})$ and if $\mathfrak{o}=\mathfrak{D} \cap k$, where $k$ is any field containing the maximal abelian extension of $\mathbb{Q}$ in $K$, then $S(\mathcal{O})=\mathfrak{D} \otimes S(\mathfrak{o})$, i.e. the Brauer classes in $S(\mathfrak{O})$ are those obtained from $S(\mathfrak{o})$ by extension of the scalars to $\mathfrak{O}$. A similar theorem is proved as well in the case of the Schur subgroup $S(\mathfrak{O}, \omega)$ of the quadratic Brauer group $B(\mathfrak{O}, \omega)$, where $\omega$ is an involution of $\mathfrak{D}$.
\end{abstract}

Suppose that $K$ is an algebraic number field (of finite degree over $\mathbb{Q}$ ) and let $\mathfrak{O}$ be the ring of $S$-integers of $K$ where $S$ is a set of primes of $K$ containing the infinite ones. Thus $\mathfrak{O}$ is a Dedekind domain whose field of quotients is $K$. Let $B(\mathfrak{O})$ denote the (linear) Brauer group of $\mathfrak{O}$. The Schur subgroup $S(\mathfrak{O})$ of $B(\mathfrak{D})$ consists of the Brauer classes which contain an Azumaya algebra $\Lambda$ over $\mathfrak{O}$ with the following property: there exists a finite group $G$ and a central simple direct summand $A$ of the group algebra $K G$ such that the image of the projection of $\mathcal{O} G$ in $A$ is $\Lambda$.

Let $\mathbb{Q}(\mu)$ denote the maximal cyclotomic extension of $\mathbb{Q}$ ( $\mu$ is the group of all roots of unity), contained in an algebraic closure of $K$. Denote by $K_{c}$ the intersection of $\mathbb{Q}(\mu)$ and $K$, and let $K \otimes S\left(K_{c}\right)$ be the subgroup of $B(K)$ obtained from $S\left(K_{c}\right)$ by extension of scalars. It is clear that $K \otimes S\left(K_{c}\right) \subseteq$ $S(K)$-in fact, they are equal by a theorem of Yamada [Prop. 4.6, Y]; see also [Theorem 3.4, M]). We shall prove the following integral analogue:

Theorem 1. Let $k$ be a subfield of $K$ containing $K_{c}$, and let $\mathfrak{o}=\mathfrak{O} \cap k$. Then

$$
S(\mathfrak{O})=\mathfrak{D} \otimes S(\mathfrak{o}) .
$$

Received by the editors November 16, 1988 and, in revised form, February 3, 1989.

1980 Mathematics Subject Classification (1985 Revision). Primary 20C10, 13A20; Secondary 16A16, 16A18, 16A26.

Key words and phrases. Schur subgroup, integral Brauer group, integral representations, representations of finite groups, quadratic Brauer group, Azumaya algebras, algebraic number fields.

Support by NSERC grant A8778 gratefully acknowledged. 
This effectively reduces the computation of $S(\mathfrak{O})$ to that of $S(\mathfrak{o})$, since $S(\mathfrak{o})$ and $S(\mathcal{O})$ are subgroups of $B(k)$ and $B(K)$ resp., and since the extension of scalars

$$
B(k) \rightarrow B(K)
$$

merely multiplies each Hasse invariant by the local degree [31.9, MO]. On the other hand $S(\mathfrak{o})$ has been determined in at least one special case, namely when $k$ is a purely cyclotomic extension of $\mathbb{Q}$. See [R1]. Similar remarks apply to Theorem 2 below.

We wish to prove an analogue in the quadratic case as well. Suppose that $\omega$ is an involution (possibly the identity) on $\mathfrak{O}$ (and $K$ ). The quadratic Brauer group $B(\mathcal{O}, \omega)$ is defined (see [H-T-W]) as the set of Morita equivalence classes of " $\omega$-antistructures" $(\Lambda, I, \varepsilon)$ where $\Lambda$ is an Azumaya algebra over $\mathfrak{D}$. (Thus $I$ is an $\omega$-antiautomorphism of $\Lambda, I^{2}$ is the inner automorphism with respect to the unit $\varepsilon$ of $\Lambda$, and $\left.\varepsilon \varepsilon^{I}=1\right)$. There is a forgetful homomorphism

$$
B(\mathfrak{O}, \omega) \stackrel{?}{\longrightarrow} B(\mathfrak{O})
$$

given by $[(\Lambda, I, \varepsilon)] \mapsto[\Lambda]$, whose image is known when $\mathfrak{O}$ is an unramified extension of the subring $\mathfrak{O}_{0}$ fixed by $\omega$ (see $[\mathrm{S}]$ ): it consists of the elements of order 2 in $B(\mathfrak{D})$ if $\omega=i d$, otherwise it is the kernel of the corestriction map Cor: $B(\mathfrak{O}) \rightarrow B\left(\mathfrak{O}_{0}\right)$.

Now consider the Schur subgroup $S(\mathfrak{O}, \omega)$ of $B(\mathfrak{O}, \omega)$, which is defined as follows: If $G$ is any finite group, let $\Omega$ be the canonical $\omega$-involution on $K G$, i.e. the map which is $\omega$ on $K$ and inverts the elements of $G$. Then the Schur subgroup $S(\mathfrak{D}, \omega)$ of the quadratic Brauer group $B(\mathcal{O}, \omega)$ consists of the Morita classes which contain an antistructure $(\Lambda, I, 1)$ (where $\Lambda$ is an Azumaya algebra over $\mathfrak{O}$ ) with the following property: There exists a finite group $G$ and a central simple direct summand $A$ of the group algebra $K G$ such that

(i) $A$ is stable under $\Omega$,

(ii) $\Lambda$ is the image of the projection of $\mathcal{D} G$ in $A$,

(iii) $I=\left.\Omega\right|_{\Lambda}$.

Note that $\Lambda$ is stable under $\Omega$ because $A$ and $\mathcal{O} G$ both are.

Let ${ }^{*}$ denote complex conjugation and define $\widetilde{K}$ to be the subfield of $K$ of elements fixed by the composition ${ }^{*} \omega$. Let $\widetilde{\mathfrak{D}}=\mathfrak{O} \cap \widetilde{K}$.

Theorem 2. Let $k$ be a subfield of $K$ containing $\widetilde{K}$ such that $k^{\omega}=k$, and let $\mathfrak{o}=\mathfrak{O} \cap k$. Then

$$
S(\mathfrak{O}, \omega)=\mathfrak{D} \otimes S(\mathfrak{o}, \omega) .
$$

The forgetful map

$$
S(\widetilde{\mathfrak{O}}, \omega) \stackrel{?}{\rightarrow} S(\widetilde{\mathfrak{O}})
$$

is onto and

$$
\operatorname{im}(S(\mathcal{O}, \omega) \stackrel{?}{\rightarrow} S(\mathfrak{O}))=\mathfrak{O} \otimes S(\tilde{\mathfrak{D}}) .
$$




\section{THE PROOFS}

We begin with a simple lemma, which in fact holds in the more general case when $\mathfrak{O}$ is a Dedekind domain and $K / k$ is an arbitrary finite separable extension.

Lemma. Let $G$ be a finite group, and suppose that the $k$-algebra $A^{\prime}$ is a direct summand of $k G$. If the projection of $\mathfrak{O} G$ on $K \otimes A^{\prime}$ is a maximal order, then the projection of oG on $A^{\prime}$ is also maximal.

Proof. Suppose that $M^{\prime}$ is an order in $A^{\prime}$ which contains the projection $\Lambda^{\prime}$ of $\mathfrak{o} G$. The projection of $\mathfrak{O} G$ on $K \otimes A^{\prime}$ is the $\mathfrak{D}$-lattice $\mathfrak{O} \Lambda^{\prime}$ generated by $\Lambda^{\prime}$. And the $\mathfrak{O}$-lattice $\mathfrak{O} M^{\prime}$ spanned by $M^{\prime}$ is obviously an $\mathfrak{O}$-order which contains $\mathfrak{O} \Lambda^{\prime}$, and so $=\mathfrak{D} \Lambda^{\prime}$. It is well known that there is a basis $\left\{x_{i}\right\}$ of $A^{\prime}$ and ideals $\mathfrak{a}_{1}, \ldots, \mathfrak{a}_{n}, \mathfrak{b}_{1}, \ldots, \mathfrak{b}_{n}$ in $\mathfrak{o}$ such that $M^{\prime}$ is the direct sum of the $\mathfrak{a}_{i} x_{i}$ and $\Lambda^{\prime}$ is the direct sum of the $\mathfrak{a}_{i} \mathfrak{b}_{i} x_{i}$. Since $\mathfrak{O} M^{\prime}=\mathfrak{O} \Lambda^{\prime}$, it follows that $\mathfrak{b}_{1}=\mathfrak{b}_{2}=\cdots=\mathfrak{o}$, and so $M^{\prime}=\Lambda^{\prime}$.

We now begin the proof of Theorem 1. Suppose that $\Lambda$ is in $S(\mathcal{O})$, arising, say, from the central simple direct summand $A$ of $K G$. Let $\chi$ be the absolutely irreducible character which belongs to $A$, and let $A^{\prime}$ be the simple summand of $k G$ corresponding to $\chi$. Since the center of $A$ is $K=K(\chi)$ (Exercise 9.15, [I]), the values of $\chi$ lie in $K$, and so also in $K \cap \mathbb{Q}(\mu) \subseteq k$. Thus $A^{\prime}$ is also central and $A=K \otimes A^{\prime}$. It follows that $\Lambda=\mathfrak{D} \otimes \Lambda^{\prime}$ where $\Lambda^{\prime}$ is the projection of $o G$ on $A^{\prime}$, and so by the above theroem $\Lambda^{\prime}$ is also a maximal order. We claim that $\Lambda^{\prime}$ is actually an Azumaya algebra. By 6.33 and 6.34 in [O-S], it suffices to prove it is split at every prime $\mathfrak{p}$ of $\mathfrak{o}$. Let $\mathfrak{P}$ be a prime of $\mathfrak{O}$ lying above $\mathfrak{p}$. Then

$$
\Lambda_{\mathfrak{P}}=\mathfrak{O}_{\mathfrak{P}} \otimes \Lambda_{\mathfrak{p}}^{\prime}
$$

where the subscripts $\mathfrak{p}$ and $\mathfrak{P}$ denote completion. We must show that the fact that $\Lambda_{\mathfrak{P}}$ is isomorphic to a full matrix algebra over $\mathfrak{D}_{\mathfrak{P}}$ implies that $\Lambda_{\mathfrak{p}}^{\prime}$ is isomorphic to one over $o_{\mathfrak{p}}$. Indeed were this not the case, the discriminant $d\left(\Lambda_{p}^{\prime}\right)$ would be properly contained in $o_{p}$ (see 20.3 and 20.4, [MO]). By (1) the discriminant of $\Lambda_{\mathfrak{P}}$ is $\mathfrak{O}_{\mathfrak{P}} d\left(\Lambda_{\mathfrak{p}}^{\prime}\right)$ which is properly contained in $\mathfrak{O}_{\mathfrak{P}}$. Contradiction. (This can also be deduced from the above lemma, and the theorem in $[\mathrm{J}])$.

It follows that $\Lambda^{\prime}$ is also an Azumaya algebra, and since

$$
\Lambda=\mathfrak{O} \otimes \Lambda^{\prime},
$$

we get the desired result at once.

We now turn to the proof of Theroem 2. Suppose that a Morita class in $S(\mathfrak{D}, \omega)$ contains the antistructure $(\Lambda, I, 1)$ arising from $\mathfrak{D} G$ as described earlier. Then $\Lambda$ is an order in the central simple $K$-algebra $A$ belonging to the absolutely irreducible character $\chi$, say. As in the proof of Theorem 1 , the values of $\chi$ lie in $K_{c}$. But in fact we can show that the values lie in $\widetilde{K}$ as 
follows (cf. the proof of Lemma 1 in [R2]). The idempotent $e_{\chi}$ of $A$ is given by the formula

$$
e_{\chi}=\frac{n}{g} \sum_{s \in G} \chi\left(s^{-1}\right) s
$$

where $n=\chi(1)$ and $g$ is the order of $G$. Since $A$ is stable under $\Omega, e_{\chi}$ is fixed by $\Omega$, whence

$$
\sum_{s \in G} \chi\left(s^{-1}\right)^{\omega} s^{-1}=\sum_{s \in G} \chi\left(s^{-1}\right) s .
$$

Using $\chi(s)=\chi\left(s^{-1}\right)^{*}$ to transform the sum on the right side, we see that $\chi\left(s^{-1}\right)^{* \omega}=\chi\left(s^{-1}\right)$ for all $s$ in $G$, and so the values of $\chi$ do indeed lie in $\widetilde{K}$, and so also in $k$.

If $A^{\prime}$ is the corresponding simple direct summand $A^{\prime}$ of $k G$, it follows that it is also central. Let $\Lambda^{\prime}$ be the projection of o $G$ on $A^{\prime}$. By the same argument as in Theoem 1, $\Lambda^{\prime}$ is an Azumaya algebra.

$A^{\prime}$ is stable under $\Omega^{\prime}$ since $A=K \otimes A^{\prime}$. Since $\Lambda^{\prime}$ is spanned over $o$ by the projections in $A^{\prime}$ of the elements of $G$, it also is stable under $\Omega^{\prime}$. If $I^{\prime}$ is the restriction of $\Omega^{\prime}$ to $\Lambda^{\prime}$, we see that

$$
(\Lambda, I)=\mathfrak{O} \otimes\left(\Lambda^{\prime}, I^{\prime}\right) .
$$

This shows that $S(\mathfrak{O}, \omega) \subseteq \mathfrak{D} \otimes S(\mathfrak{o}, \omega)$, in fact that

$$
S(\mathfrak{O}, \omega)=\mathfrak{O} \otimes S(\mathfrak{o}, \omega) .
$$

Let $\widetilde{\Omega}$ be the canonical $\omega$-involution on $\widetilde{K} G$ and consider the pairing $\frac{1}{g} T\left(x y^{\widetilde{\Omega}}\right)$ of $\tilde{K} G$ into $\widetilde{K}$ where $T$ is the ordinary algebra trace. Since $\omega=^{*}$ on $\widetilde{K}$, it is easy to see that this pairing is an hermitian form with respect to complex conjugation. Moreover, $G$ forms an orthonormal basis and so the form is positive definite; thus $\frac{1}{g} T\left(x x^{\widetilde{\Omega}}\right)>0$ for all $x \neq 0$ in $\widetilde{K} G$, and it follows at once that every simple direct summand of $\widetilde{K} G$ is stable under $\widetilde{\Omega}$. Therefore the projection of $\widetilde{\mathfrak{D}} G$ on such a summand is also stable, whence

$$
S(\widetilde{\mathfrak{O}}, \omega) \rightarrow S(\widetilde{\mathfrak{O}})
$$

is onto. We then obtain

$$
\operatorname{im}(S(\mathfrak{O}, \omega) \rightarrow S(\mathfrak{O}))=\mathfrak{O} \otimes S(\widetilde{\mathfrak{D}})
$$

by application of (2) in the case $k=\widetilde{K}$.

\section{REFERENCES}

[H-T-W] I. Hambleton, L. Taylor, and E. B. Williams, An introduction to the maps between surgery obstruction groups, Algebraic Topology, Aarhus 1982, pp. 49-127; Lecture Notes in Math., vol. 1051, Springer-Verlag, New York, 1984.

[I] I. M. Isaacs, Character theroy of finite groups, Academic Press, New York, 1976.

[J] G. J. Janusz, Tensor products of orders, J. London Math. Soc. (2) 20 (1979), 186-192.

[M] R. Mollin, The Schur group of a field of characteristic zero, Pacific J. Math. 76 (1978), 471-478. 
[MO] I. Reiner, Maximal orders, London Math. Soc. Monographs, no. 5, Academic Press, London, 1975.

[O-S] M. Orzech and C. Small, The Brauer group of commutative rings, Lecutre Notes in Pure and Appl. Math., no. 11, Dekker, New York, 1975.

[R1] C. Riehm, The Schur subgroup of the Brauer group of cyclotomic rings of integers, Proc. Amer. Math. Soc. 103 (1988), 27-30.

[R2] C. Riehm, The quadratic Schur subgroup over local and global fields Math. Annalen, 283 ((1989), 479-489.

[S] D. Saltman, Azumaya algebras with involution, J. Algebra 52 (1978), 526-539.

[Y] T. Yamada, The Schur subgroup of the Brauer group, Lecture Notes in Math., vol. 397, Springer-Verlag, New York, 1974.

Department of Mathematics and Statistics, McMaster University, Hamilton, Ontario L8S 4K1, Canada 